\title{
Fundamentals of differential particle inertial focusing in symmetric sinusoidal microchannels
}

Jun Zhang ${ }^{\mathrm{a} *}$, Dan Yuan ${ }^{\mathrm{b}}$, Qianbin Zhao ${ }^{\mathrm{b}}$, Adrian J.T. Teo ${ }^{\mathrm{a}}$, Sheng Yan ${ }^{\mathrm{c}}$, Weihua $\mathrm{Li}^{\mathrm{b}}$, NamTrung Nguyen ${ }^{\mathrm{a} *}$

${ }^{\text {a }}$ Queensland Micro and Nanotechnology Centre, Griffith University, Brisbane QLD 4111, Australia, Fax: +61 07373 58021; Tel: +61 (07) 373 53921; E-mail: jun.zhang@griffith.edu.au; nam-trung.nguyen@griffith.edu.au

b School of Mechanical, Materials and Mechatronic Engineering, University of Wollongong, Wollongong, NSW 2522, Australia

c Department of Chemistry, University of Tokyo, Tokyo, 113-0033, Japan

\begin{abstract}
:
Focusing and separation of particles such as cells at a high throughput are extremely attractive for biomedical applications. Particle manipulation based on inertial effects requires a high flow speed, and thus suits well to high-throughput applications. Recently, inertial focusing and separation using curvilinear microchannels have been attracting a great interest due to the linear structure for parallelization, small device footprint, superior particle focusing performance and easy implementation of particle separation. However, the curvature direction of these microchannels is alternating, leading to the variation in both the magnitude and direction of the induced secondary flow. The accumulation of this variation along the channel causes unpredictable behaviour of particles. This paper systematically investigates the inertial focusing phenomenon in low-aspect-ratio symmetric sinusoidal channels. First, we comprehensively studied the effects of parameters such as viscosity, flow condition, particle size, and geometric dimension of microchannel on differential particle focusing. We found that particle inertial focusing is generally independent of fluid kinematic viscosity, but highly dependent on particle size, flow condition and channel dimension. Next, we derived an explicit scaling factor and included all four dimensionless parameters (particle blockage ratio, curvature ratio and Dean number, and channel aspect ratio) in a single operational map to illustrate the particle focusing patterns. Finally, we proposed a rational guideline to intuitively instruct the design of channel dimensions for separation of a given particle mixture.
\end{abstract}

\section{Introduction}


Continuous focusing and separation of microparticles are indispensable for a wide range of biomedical applications. In terms of microparticle manipulation, microfluidic technology with characteristic channel dimension on the order of tens to hundreds of micrometres provides superior advantages over conventional large-scale platforms ${ }^{1-8}$. Among a variety of available microfluidic separation technologies, focusing and separation of microparticles based on hydrodynamic inertial effects are extremely attractive for high-throughput applications due to its simplicity, precision, robustness and high flow rate on the order of millilitres per minute ${ }^{8-10}$. The high throughput is especially beneficial for large-volume sample processing and isolation of bioparticles with low abundance such as circulating tumour cells (CTCs) ${ }^{11}$.

To date, focusing and separation of microparticles using inertial microfluidics have demonstrated a range of successful biomedical applications such as extraction of blood plasma ${ }^{12}$, separation of particles and cells ${ }^{13,14}$, solution exchange ${ }^{15,16}$, cell enrichment ${ }^{17}$, isolation of CTCs ${ }^{18-20}$, detection of malaria pathogen ${ }^{21}$, microfiber fabrication ${ }^{22}$, cell cycle synchronization ${ }^{23}$, cell encapsulation ${ }^{24}$ and single cell stretching ${ }^{25}$. Accurate prediction of fluid and microparticle motion in the suitably structured microchannels is essential for the success of the above applications. However, the determination of microparticle behaviours and the optimisation of microchannel geometry were all based on the time-consuming and laborious experimental trials, and only for a specific particle mixture. The lack of a practical design guide delays the design and testing stage, inhibiting further applications of inertial microfluidics.

Recently, inertial microfluidics based on curvilinear channel design has been attracting increasing interests due to its promising potential in parallelization, superior particle focusing performance and easy implementation of particle separation. Wang and Dandy ${ }^{26}$ developed a microfluidic system with asymmetric curvilinear channels with an aspect ratio or height to width ratio of $H / W=1 / 2$ to focus and concentrate sub-micrometre particles. Successful focusing of sub-micrometre particles and cyanobacteria demonstrated the suitability of inertial microfluidics for manipulating bacteria and subcellular organelles. Zhou et al. ${ }^{27}$ successfully demonstrated the separation of MCF-7 cancer cells from diluted whole blood in a serial reverse wavy microchannel. Meanwhile, fundamental works exploring inertial focusing phenomenon in curvilinear channels have also been reported. Di Carlo et al. ${ }^{28}$ have conducted pioneering works on investigation of alternating curvatures on inertial migration of 
particles in curvilinear channels. In their study, the four equilibrium positions in a straight channel with a square cross-section were reduced to two positions in a symmetric sinusoidal channel. These focusing positions could further reduce to a single position using asymmetric curvatures. They proposed for the first time the scaling ratio of inertial lift force to Dean drag $F_{L} / F_{D^{\sim}} 2 \mathrm{ra}^{2} / \mathrm{D}_{h}{ }^{3}$, by assuming that the shear gradient lift balances Dean drag. Besides, a state diagram of particle focusing in curved channels was developed as a function of force ratio and channel Reynolds number, which could serve as design criteria for the focusing of potentially arbitrary-sized particles ${ }^{29}$. Although this map provides a general trend on the particle focusing state, the boundary and region of each state is not clear, thus it is not easy to design channels for particle separation based on the map. One possible reason is that the expression of shear gradient lift force was derived from the square channels. However, in asymmetric serpentine channels, the cross section varies along the curvatures, causing errors between theoretical analysis and experimental results. Particle filtration and separation could also be achieved by tailoring the force ratio of $F_{L} / F_{D}$ for different particles, where $F_{L} / F_{D} \geq 1$ for a particle of a given size and $F_{L} / F_{D}<1$ for a particle of another size. For $F_{L} / F_{D} \geq 1$, inertial lift forces that push particles to an equilibrium position dominate, while for $F_{L} / F_{D}<1$, Dean drag is dominant and leads to mixing of particles ${ }^{30}$. Our previous works 31,32 demonstrated that both two-position and single-position focusing patterns could occur in symmetric serpentine microchannels with a low aspect ratio. We further demonstrated the complete separation of particles and biological cells using differential focusing positions ${ }^{33,34}$. Due to their linear nature, serpentine channels can be easily parallelized to amplify the whole throughput in a small footprint ${ }^{31}$. The above preliminary results indicate that symmetric curvilinear channels are promising candidates for the separation and enrichment of various bioparticles.

However, there are still critical challenges to be addressed to pave the way for wide applications of curvilinear channels for filtration and separation of bioparticles. The challenges include: (i) the unknown influence of channel geometry and size on particle inertial focusing; (ii) the unknown role of the fluid viscosity on inertial focusing; (iii) the lack of suitable metric for predicting focusing location of particles in the curvilinear microchannels; and (iv) the lack of a practical guide on microchannel design for sorting a specific particle mixture. Therefore, our present work aims to systematically investigate the effects of particle size relative to channel size, channel geometry, fluid viscosity and flow condition on particle inertial focusing. By examining the influences of fluid kinematic viscosity and four dimensionless parameters (particle blockage ratio, curvature ratio, Dean 
number and channel aspect ratio), we found that particle inertial focusing is independent of fluid kinematic viscosity but highly related to particle size relative to channel size, channel geometry, channel aspect ratio and flow condition. Furthermore, we derived a scaling parameter based on the four dimensionless parameters and generalised our experimental data in a single operational map for the different regimes of particle focusing in symmetric sinusoidal microchannels. Finally, we proposed for the first time a rational design guide of sinusoidal microchannels for separation of a given particle mixture.

\section{Theory}

\subsection{Inertial migration}

Inertial migration is a phenomenon where randomly dispersed particles at the entrance of a channel migrate laterally to several cross-sectional equilibrium positions after a long enough passage ${ }^{35,36}$. Inertial migration occurs in the intermediate flow regime $(\sim 1<\operatorname{Re}<\sim 100$,) between the Stokes flow regime $(\operatorname{Re} \rightarrow 0$, ) and the turbulent flow regime $(\operatorname{Re}>\sim 2000)$. The Reynolds number $\operatorname{Re}=\rho U D_{\mathrm{h}} / \mu$, as the ratio of inertial forces to viscous forces within a fluid, is used to distinguish these regimes, where $\rho, U$ and $\mu$ are fluid density, average velocity and dynamic viscosity respectively. The hydraulic diameter $D_{\mathrm{h}}$ of the channel is defined as $D_{\mathrm{h}}=D$ for a circular channel with $D$ the diameter of the circular cross-section or $D_{\mathrm{h}}=2 \mathrm{WH} /(\mathrm{W}+H)$ for a rectangular channel with $W$ and $H$ the width and height of the cross-section. Particle inertial migration in a straight channel is widely recognized as the counteraction of two inertial forces: the shear gradient lift force $F_{L S}$ and the wall lift force $F_{L W}$. The shear gradient lift force $F_{\text {LS }}$ originates from the parabolic flow velocity profile and its interaction with the finite particle size, directing toward the channel wall. The wall lift force $F_{L W}$ arises from the asymmetric vortices around the particles when they approach the channel wall, pointing toward channel centerline. Assuming that the particle is much smaller than the channel dimension, the net inertial lift force $F_{L}$ is expressed as ${ }^{8,37}$ :

$$
F_{\mathrm{L}}=\frac{\rho U^{2} \mathrm{a}^{4}}{\mathrm{D}_{\mathrm{h}}^{2}} f_{\mathrm{L}}
$$

where $a$ is the particle diameter, $f_{\mathrm{L}}$ is the dimensionless coefficient of the inertial lift force, which is a function of the particle cross-sectional position, particle size and Reynolds number 8 .

\subsection{Dean flow}

In a curved channel, fluid momentum mismatch in the centre and near-wall region within the curvature generates a pressure gradient along the radial direction. Fluid near the channel 
centerline flows outwards due to the higher momentum and pushes the relatively stagnant fluid near the wall inwards along the circumference leading to two counter-rotating vortices, also called Dean vortices ${ }^{8}$. The magnitude of the rotational flow velocity scales as ${ }^{28}$ :

$$
U_{\mathrm{D}} \sim \operatorname{De}^{2} v / D_{\mathrm{h}}
$$

where $v$ is the kinematic viscosity of the fluid, $v=\mu / \rho$. The Dean number De is defined as a function of the hydraulic diameter, the radius of the channel curvature $R$ and the Reynolds number $\mathrm{Re}^{38}$ :

$$
\mathrm{De}=\sqrt{\frac{D_{\mathrm{h}}}{2 R}} \operatorname{Re}
$$

Assuming the Stokes law and that the particle is kept in a stationary cross-sectional position, the magnitude of the Dean drag force $F_{D}$ scales as ${ }^{13}$ :

$$
F_{\mathrm{D}} \sim \frac{\mu^{2} a \mathrm{De}^{2}}{\rho D_{\mathrm{h}}}
$$

\subsection{Coupling of inertial lift forces and Dean drag force}

The coupling of inertial lift forces and Dean drag force in a curved channel could be used to modify inertial focusing and the equilibrium position of the particles ${ }^{28}$. The relative ratio between the inertial lift force and the Dean drag force is ${ }^{8}$ :

$$
\frac{F_{\mathrm{L}}}{F_{\mathrm{D}}} \sim \frac{\mathrm{R}_{\mathrm{e}}^{2}}{\mathrm{De}^{2}}\left(\mathrm{a} / D_{\mathrm{h}}\right)^{3} f_{\mathrm{L}}
$$

Furthermore, the recent work of Zhou and Papautsky 39 suggested the relationship $f_{\mathrm{L}} \sim \frac{1}{\sqrt{\mathrm{Re}}}\left(\frac{D_{h}}{\mathrm{a}}\right)^{2}$, therefore:

$$
\frac{\mathrm{F}_{\mathrm{L}}}{\mathrm{F}_{\mathrm{D}}} \sim \frac{\left(\mathrm{a} / \mathrm{D}_{\mathrm{h}}\right)}{\mathrm{De}^{1 / 2}\left(\mathrm{D}_{\mathrm{h}} / 2 \mathrm{R}\right)^{3 / 4}}=\delta
$$

Here, the dimensionless number $\delta$ is an indicator for the ratio between the inertial lift force $F_{\mathrm{L}}$ and the Dean drag force $F_{\mathrm{D}}$. This number depends on the channel curvature ratio $\left(D_{\mathrm{h}} / 2 R\right)$, the Dean number (De) and the particle blockage ratio $\left(a / D_{\mathrm{h}}\right)$ or the relative size of particle to channel. Ideally, the value of $\delta$ should directly correspond to the regime of particle focusing in curved microchannels. However, the exact threshold for different focusing patterns is still unknown to date. It is still unclear whether its value is universal and independent of channel geometric parameter, flow parameter or particle dimension. For simplicity, our present work only focuses on symmetric sinusoidal microchannels, so that the channel cross-section and curvature radius remain unchanged for each channel. We will explore the effects of dimensionless parameters - channel curvature ratio $\left(D_{\mathrm{h}} / 2 R\right)$, Dean 
Number (De), particle blockage ratio $\left(a / D_{\mathrm{h}}\right)$ and aspect ratio $(H / W)$ on particle differential focusing patterns. We will propose an operational map which explicitly relates these dimensionless parameters to the differential focusing patterns of particles.

\section{Results and discussion}

\subsection{Effect of Dean number De}

Low-aspect-ratio microchannels are commonly used in microfluidics because the relatively wide and shallow channels can be easily fabricated. The features of the microchannels remain intact during the de-moulding process. The present work investigates inertial focusing in symmetric sinusoidal microchannels with a low aspect ratio $(H / W \leq 1 / 2)$, Figure $1(a)$.

We introduced a solution with $15-\mu \mathrm{m}$ particles into one typical sinusoidal microchannel ( $W=200 \mu \mathrm{m}, H=50 \mu \mathrm{m}, R=250 \mu \mathrm{m}$ ) with a flow rate ranging from $200 \mu \mathrm{l} / \mathrm{min}$ to $2,000 \mu \mathrm{l} / \mathrm{min}$, with corresponding Dean number ranging from 10.7 to 107 . The particle trajectories at the end of the serpentine channels were captured using a high-speed camera. The particle position and occurrence frequency along the channel width were analysed and plotted in a colour-coded map, Figure 1(b). The colour in the map indicates the normalized frequency of the particles at a specific lateral position. Generally, the pattern of particle positions with increasing flow rate assumes a dumbbell shape. At a relatively low flow rate, particles are focused as two streaks along the sidewalls, Figure 1(c-i). Increasing the flow rate leads to two focused streaks shifting toward the channel centreline, Figure 1(c-ii), and finally combining as a single streak at the centre when the flow rate is above a threshold, Figure 1(c-iii). Further increasing the flow rate maintains the single-position focusing pattern until the flow rate is above a second threshold, the focused streak starts to defocus again as a wide band due to the strong mixing effect of the secondary flow, Figure 1(c-iv). 
(a)

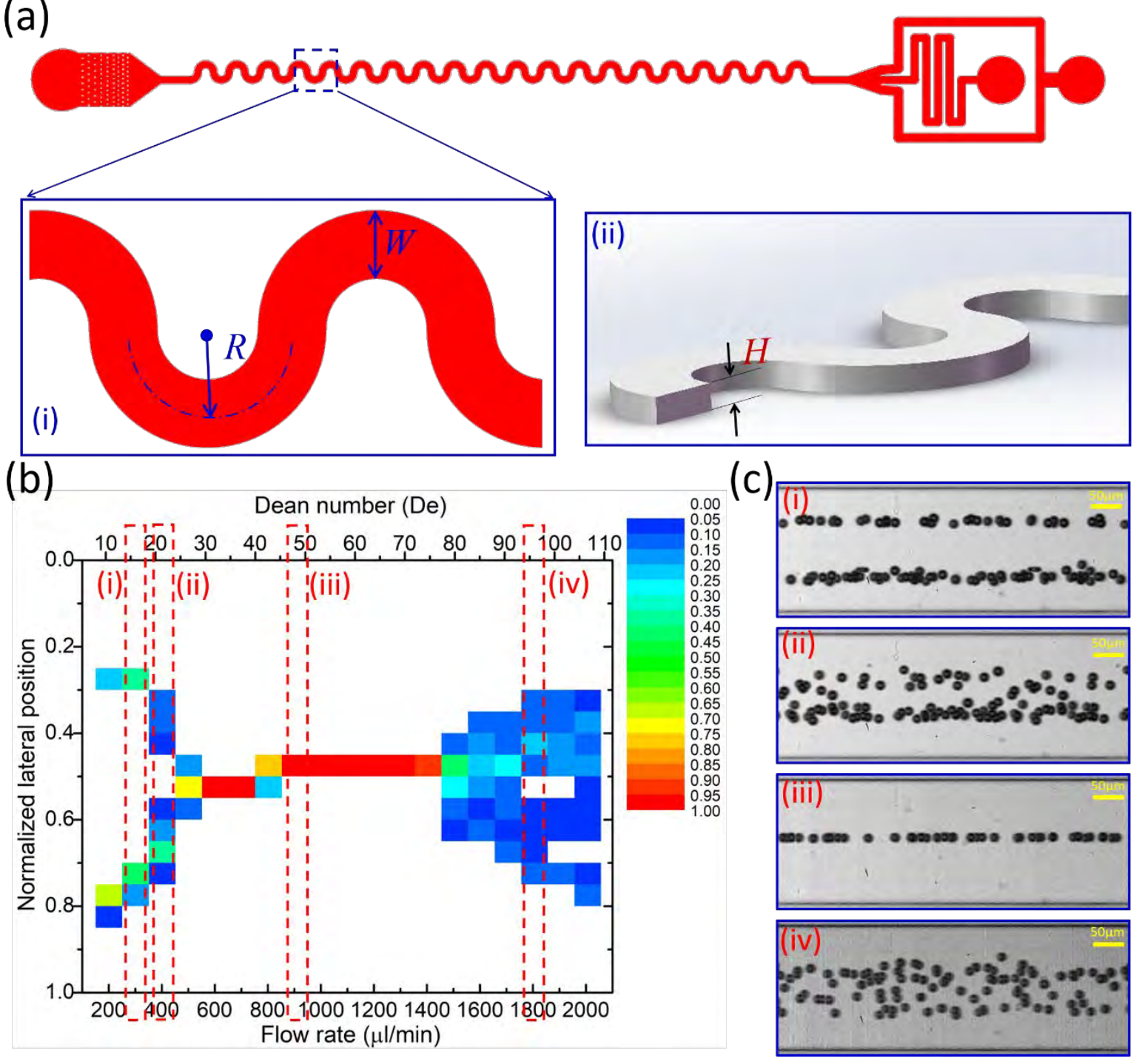

Figure 1 (a) Geometric parameters of symmetric sinusoidal microchannels. (b) The colourcoded map illustrates particle inertial focusing in symmetric sinusoidal microchannels ( $W=200 \mu \mathrm{m}, H=50 \mu \mathrm{m}, R=250 \mu \mathrm{m}$ ) at different flow conditions (flow rate or Dean number). For each flow condition, a high-speed video with 1,000 frames was processed to extract information of particle position and occurrence frequency. (c) Stacked high-speed bright field images at the end of the sinusoidal channels correspond to the four typical regions of (i) twoposition focusing, (ii) transition, (iii) single-position focusing, and (iv) defocusing in (b).

\subsection{Effect of particle blockage ratio $a / D_{h}$}

Particle size relative to the channel size is a critical parameter for successfully focusing particles in microchannels. The particle blockage ratio $a / D_{\mathrm{h}}$ is the size ratio of the particle and 
the microchannel. We investigated the influence of particle blockage ratio $a / D_{\mathrm{h}}$ on the different focusing patterns. Five sets of polystyrene beads (5 $\mu \mathrm{m}, 10 \mu \mathrm{m}, 13 \mu \mathrm{m}, 15 \mu \mathrm{m}$, and $20 \mu \mathrm{m}$ in diameter) were tested with flow rates from $200 \mu 1 / \mathrm{min}$ to $2,000 \mu 1 / \mathrm{min}$ in three symmetric sinusoidal microchannels. The focusing characteristics under different Dean numbers De were plotted as colour-coded maps in Figures 2(a)-(c). In general, the particle inertial focusing patterns and their evolution for different particle dimensions are similar. The two-position focused streaks shift inward to the centreline and merge into a single focused streak for a particular Dean number range. As the Dean number further increases, the single focused streak scatters into a more complicated pattern. However, there are obvious differences between particles of different sizes. The focusing regimes are determined by a critical Dean number $\left(\mathrm{De}_{\mathrm{c}}\right)$, at which the initial two-position pattern starts to morph into the single-position pattern. The critical Dean number $\left(\mathrm{De}_{\mathrm{c}}\right)$ is defined as the lowest value of the Dean number, where two focused branches merge into a single but relatively wide band of particles, which is the transition region between the two-position pattern and single-position pattern. Figure 2(d) shows that the larger the particle blockage ratio, the lower is the critical Dean number $\mathrm{De}_{\mathrm{c}}$ for the transition. However, this relationship seems to contradict with the scaling relationship of equation (6), if the value of $\delta$ is assumed to be constant for the occurrence of the transition region. According to equation (6), $\delta \sim\left(a / D_{\mathrm{h}}\right) / \mathrm{De}^{1 / 2}$, a larger particle blockage ratio requires a higher Dean number De to keep $\delta$ constant. This contradiction implies that the value of $\delta$ is highly dependent on particle blockage ratio for different focusing patterns, which will be verified later.

Examining the lateral distribution of particles with different particle blockage ratios more closely indicates that the width of the focused streaks of small particles is wider than that of large particles for the same De. For example, the streak width of small particles of $a / D_{\mathrm{h}}=0.0625$ is about 8 times of the particle diameter for De ranging from 10 to 35, but it is only about 1 to 2 times the diameter of larger particles of $a / D_{\mathrm{h}}=0.125,0.1625$ and 0.25 , Figure 2(a). Furthermore, the lateral positions of the focused streaks are statistically closer to the channel centreline for larger particles than the smaller ones at the same De, enabling possible particle separation by differential lateral positions. Moreover, the De range for twoposition pattern shrinks with increasing particle size. In contrast, the De range for singleposition pattern expands with increasing particle size.

We also investigated the effects of medium on particle inertial focusing in symmetric serpentine channels. The critical Dean number $\mathrm{De}_{\mathrm{c}}$ is generally independent of kinematic 
viscosity of the medium when the glycerol volume ratio is low $(\leq 20 \%)$, and more complex phenomenon appear for glycerol volume ratio $\geq 30 \%$, Figure S2.
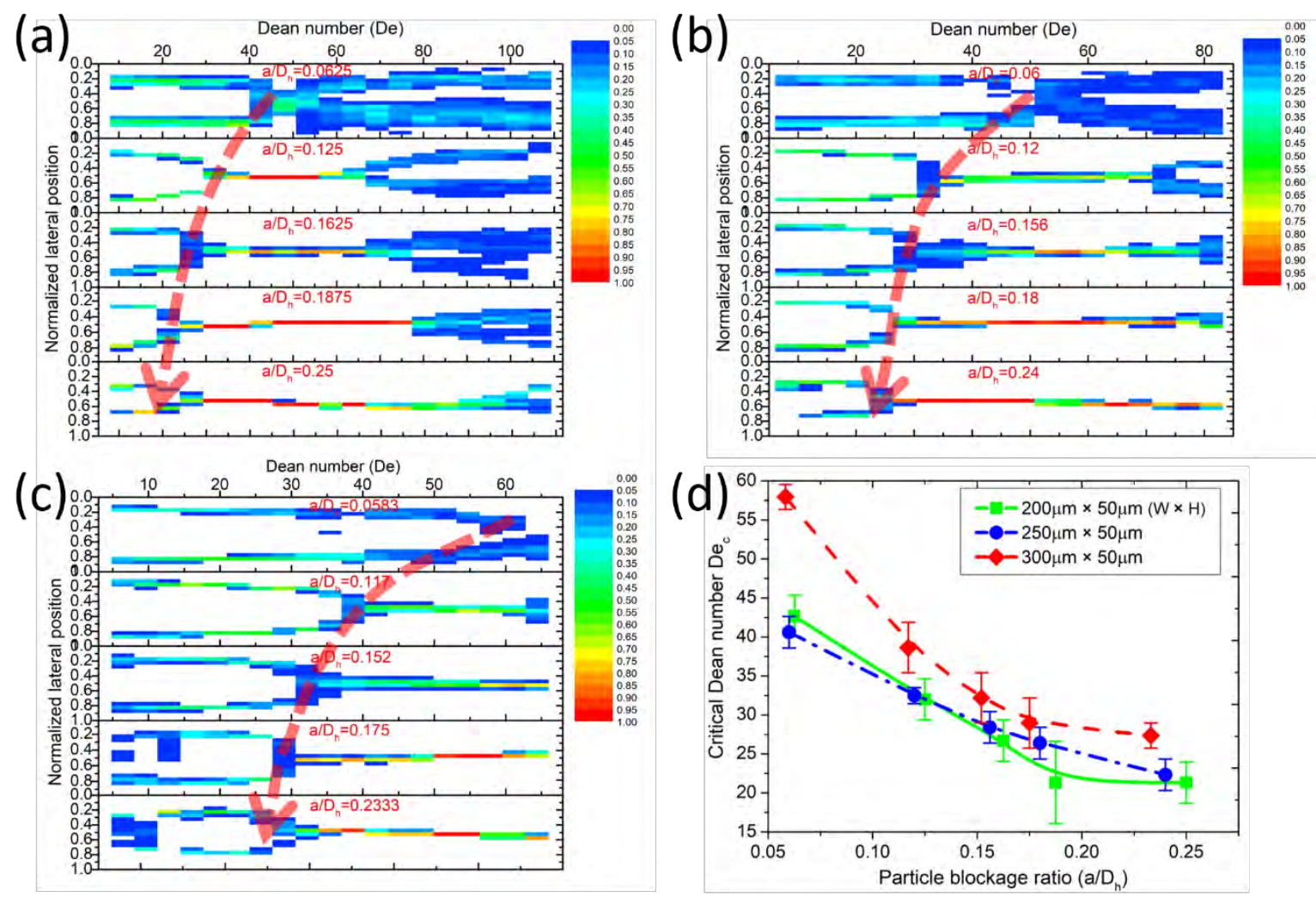

Figure 2 Inertial focusing of polystyrene beads with different blockage ratio $\left(a / D_{\mathrm{h}}\right)$ in symmetric serpentine channels with cross sections of (a) $200 \mu \mathrm{m} \times 50 \mu \mathrm{m} \times 250 \mu \mathrm{m}(W \times H \times R)$, $D_{\mathrm{h}} / 2 R=0.16$, (b) $250 \mu \mathrm{m} \times 50 \mu \mathrm{m} \times 312 \mu \mathrm{m}(W \times H \times R), D_{\mathrm{h}} / 2 R=0.1335$, (c) $300 \mu \mathrm{m} \times 50 \mu \mathrm{m} \times 375$ $\mu \mathrm{m}(W \times H \times R), D_{\mathrm{h}} / 2 R=0.1143$. (d) The relationship between the critical Dean number and the particle blockage ratio. The critical Dean number $\left(\mathrm{De}_{\mathrm{c}}\right)$ is defined as the lowest Dean number De where two-position focused pattern disappears and a wide distribution of particles arises, which is a transition region to single-position focused pattern.

\subsection{Influence of channel curvature ratio $D \mathfrak{h} / 2 R$}

The channel curvature ratio $\left(D_{\mathrm{h}} / 2 R\right)$ is a geometric parameter that considers the size of channel cross-section and channel curvature. The larger the curvature ratio, the stronger is the secondary flow for the same Reynolds number Re. In our experiments, the particle blockage ratio $\left(a / D_{\mathrm{h}}\right)$ was kept constant. The colour-coded maps of inertial focusing patterns with increasing channel curvature ratios $\left(D_{\mathrm{l}} / 2 R\right)$ for three sets of particle blockage ratios are depicted in Figures 3(a)-(c). The critical Dean number $\mathrm{De}_{\mathrm{c}}$ decreases with increasing 
curvature ratio $\left(D_{\mathrm{h}} / 2 R\right)$. Furthermore, the experimental data indicate a linear relationship between the critical Dean number $D_{\mathrm{c}}$ and the channel curvature ratio $D_{\mathrm{l}} / 2 R$, Figure 3(d). This linear relationship agrees qualitatively well with the scaling relationship of equation (6), where $\delta \sim 1 /\left[\mathrm{De}^{1 / 2}\left(D_{\mathrm{h}} / 2 R\right)^{3 / 4}\right]$, by taking in consideration of the particle size deviation and the fabrication error of the microchannels.
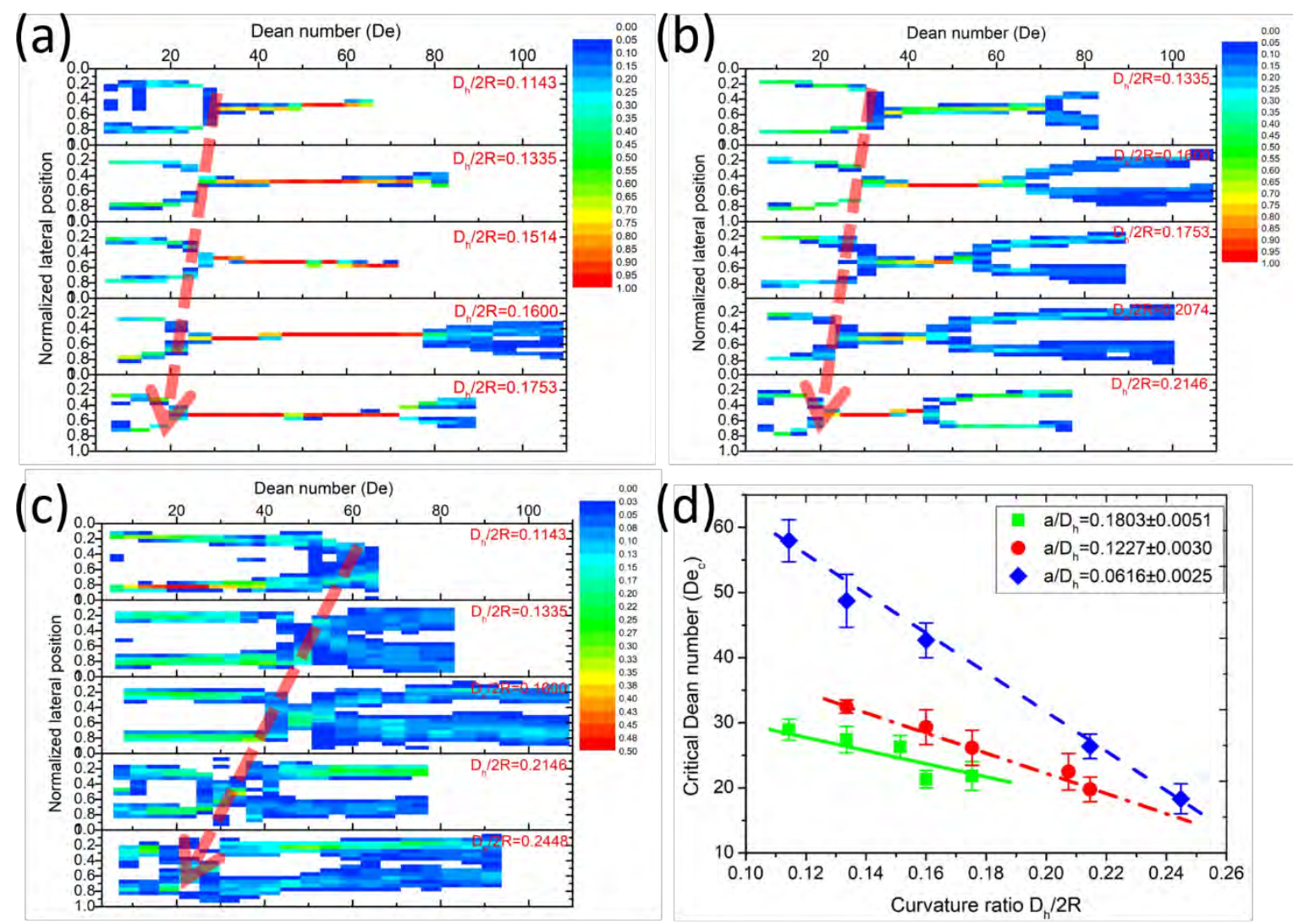

Figure 3 Effects of curvature ratio $\left(D_{\mathrm{h}} / 2 R\right)$ on particle inertial focusing when (a) $a / D_{\mathrm{h}}=0.1803 \pm 0.0051 ; \quad$ (b) $a / D_{\mathrm{h}}=0.1227 \pm 0.0030 ;$ and (c) $a / D_{\mathrm{h}}=0.0616 \pm 0.0025$. (d) The relationship of critical Dean number $D e_{c}$ with channel curvature ratio $D_{\mathrm{h}} / 2 R$. De $e_{\mathrm{c}}$ decreases almost linearly with increasing $D_{\mathrm{h}} / 2 R$.

\subsection{Effect of channel aspect ratio $H / W$}

The channel cross-sectional shape affects the distribution of the secondary flow, and thus alters the particle focusing distribution and pattern. Based on the scaling relationship of (6), the influence of the aspect ratio $H / W$ on the force ratio $F_{\mathrm{L}} / F_{\mathrm{D}}$ is implicitly reflected in the term of channel hydrodynamic diameter $D_{\mathrm{h}}=2 \mathrm{WH} /(W+H)$. Here, we investigated the common rectangular cross-sectional shape. By keeping either width or height constant, and 
changing the other one, we investigated the influence of the channel cross-sectional shape on particle inertial focusing in symmetric sinusoidal microchannels. Figure 4 shows that increasing the channel width delays the transition from two-position focusing pattern to single-position focusing pattern, leading to a higher critical Dean number De $e_{c}$, Figures 4(a) and (c). In contrast, increasing the channel height can bring this transition forward, lowering the critical Dean number $\mathrm{De}_{\mathrm{c}}$, Figures 4(b) and (d). The aspect ratio, which is defined as the ratio of the channel height $H$ to the channel width $W$, was used to describe the cross-sectional shape of the microchannel. Obviously, Figures 4(c) and (d) show that increasing the channel aspect ratio $(H / W)$ consistently decreases the critical Dean number $\mathrm{De}_{\mathrm{c}}$. A lower $\mathrm{De}_{\mathrm{c}}$ is required for the higher aspect ratio channel to achieve the same single-position focusing. Even though a lower De represents a smaller secondary flow velocity, the secondary flow that contributes to single-position focusing is sufficiently strong in high aspect-ratio channels.

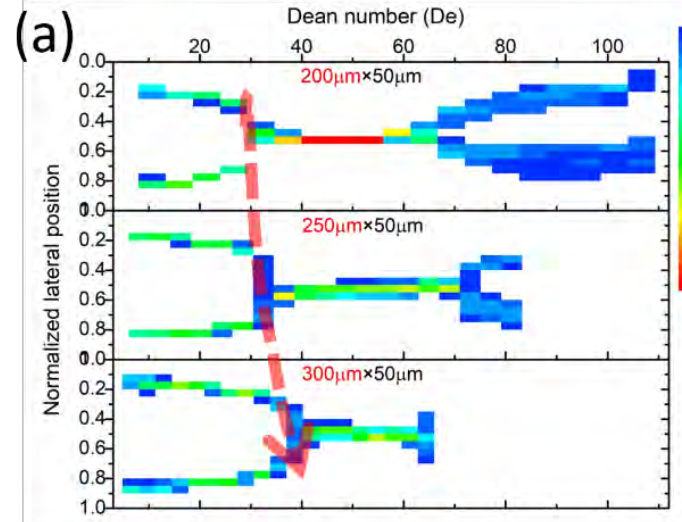

(c)

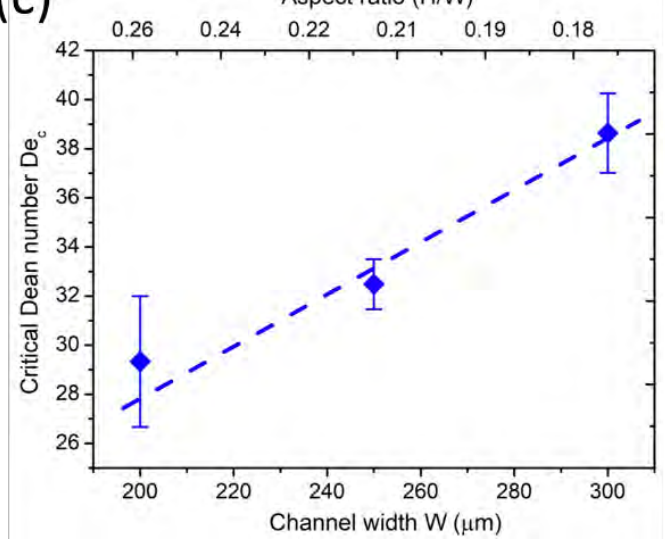

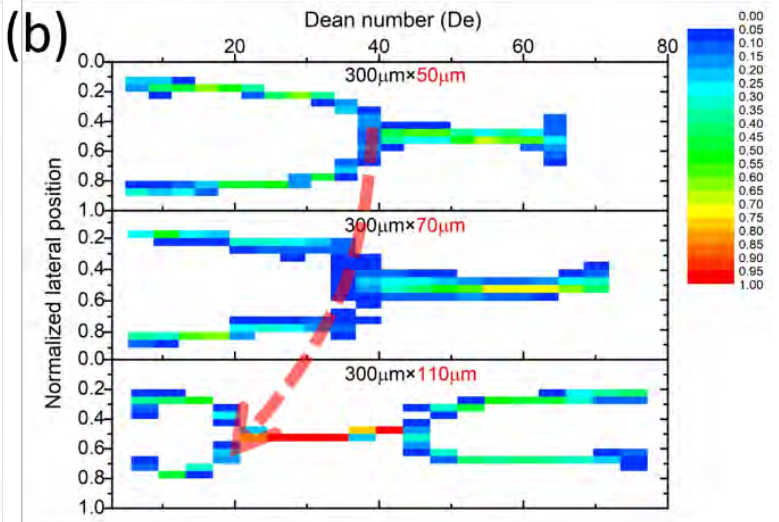

(d)

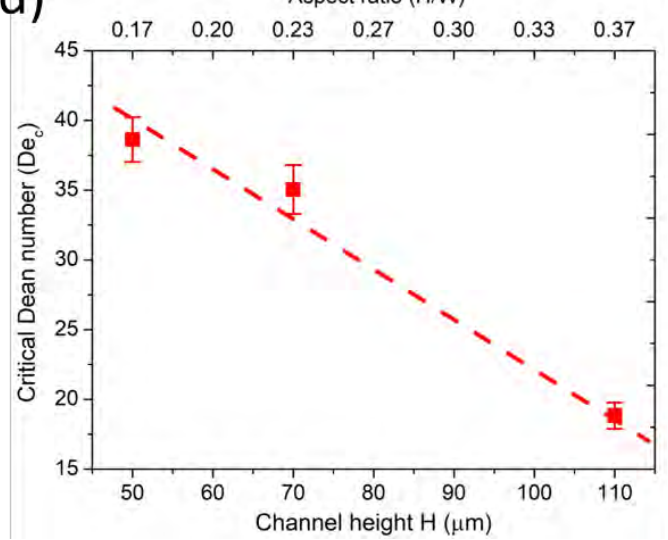

Figure 4 (a) Particle focusing in symmetric sinusoidal channels of varying channel widths with other geometric parameters remaining constant and a particle blockage ratio of $a / D_{\mathrm{h}}=0.121 \pm 0.004$. (b) Particle focusing in symmetric sinusoidal channels of varying channel height with particle blockage ratio of $a / D_{\mathrm{h}}=0.1193 \pm 0.0047$. (c) The critical Dean number $D e_{c}$ increases with the increasing channel width $W$. (d) The critical Dean number $\mathrm{De}_{\mathrm{c}}$ decreases 
with increasing channel height $H$. In both cases, the critical Dean number $\mathrm{De}_{c}$ decreases with increasing channel aspect ratio $(H / W)$.

\subsection{Differential focusing operational map}

Based on Figure 3, we calculated the $\delta$ value for three particle blockage ratios $\left(a / D_{\mathrm{h}}=0.1803 \pm\right.$ $0.0051,0.1227 \pm 0.0030$, and $0.0616 \pm 0.0025)$. We subsequently plotted particle differential focusing patterns (two-position focusing, single-position focusing and defocusing) in a map of $\delta=\left(a / D_{\mathrm{h}}\right) /\left[\mathrm{De}^{1 / 2}\left(D_{\mathrm{h}} / 2 R\right)^{3 / 4}\right]$ vs Dean number De, Figures 5(a) (c). The results indicate that for the same particle blockage ratio, the range of $\delta$ for each focusing pattern is generally constant, independent of the sinusoidal channel dimension (e.g., curvature ratio). The ranges of $\delta$ for differential focusing patterns are highly dependent on particle blockage ratio. A theoretical operational map was plotted based on the linear fitting of the three particle blockage ratios, Figure 5(d). We can observe a particle dimension threshold that particle can achieve single-position focusing and the linear extrapolation predicts that the minimum

particle block ratio $\left(a / D_{\mathrm{h}}\right)$ for particle successful single-position focusing is 0.08 , which is comparable with that $a / D_{\mathrm{h}}>0.07$ in asymmetrically curvilinear channels with a square crosssection $(H / W=1)^{28}$. 

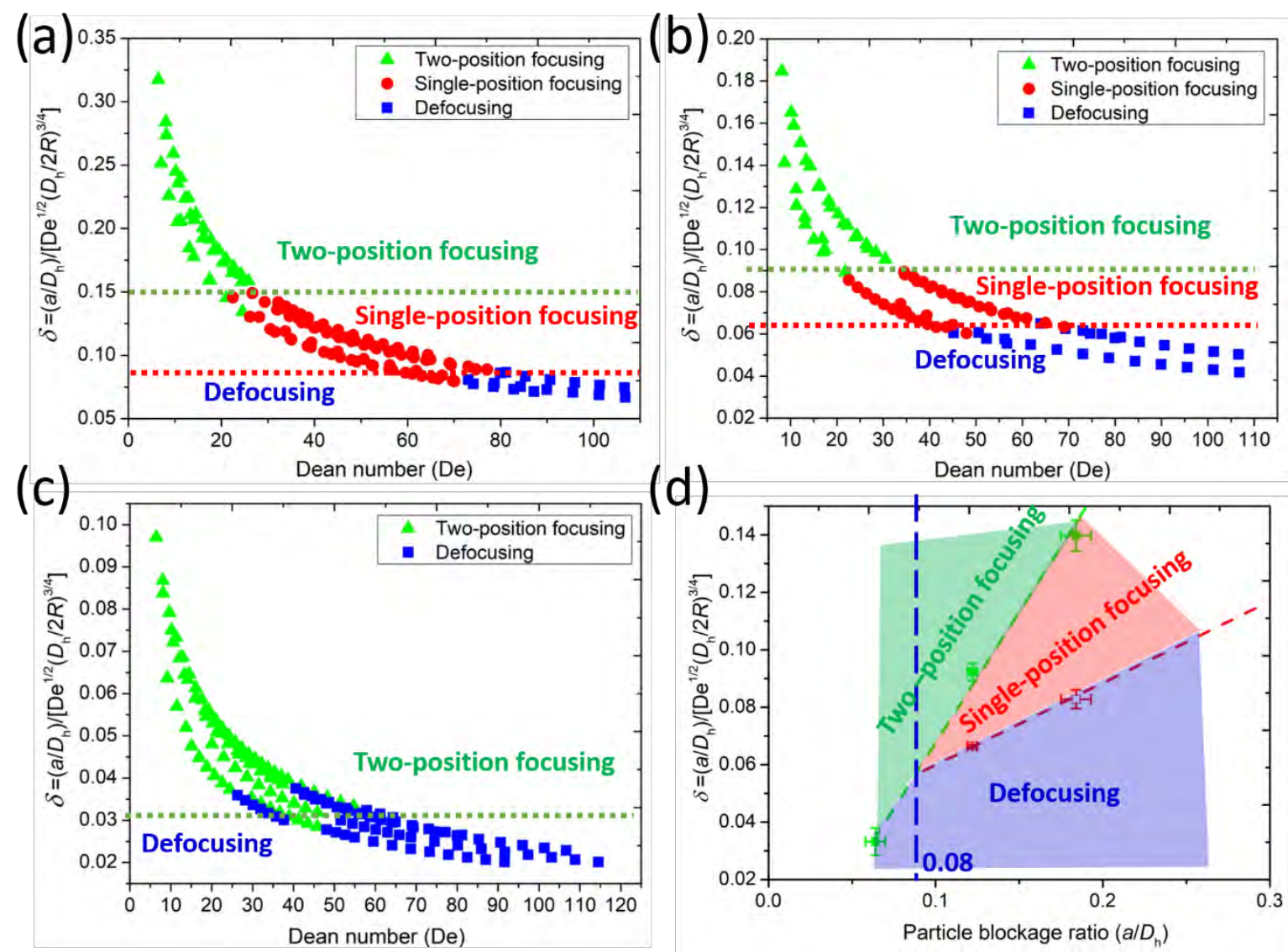

Figure 5 The magnitude of $F_{\mathrm{L}} / F_{\mathrm{D}}$ scaling factor $\delta=\left(a / D_{\mathrm{h}}\right) /\left[\mathrm{De}^{1 / 2}\left(D_{\mathrm{h}} / 2 R\right)^{3 / 4}\right]$ for differential focusing patterns when particle blockage ratio is: (a) $a / D_{\mathrm{h}}=0.1803 \pm 0.0051$; (b) $a / D_{\mathrm{h}}=0.1227 \pm$ 0.0030 ; and (c) $a / D_{\mathrm{h}}=0.0616 \pm 0.0025$. (d) The theoretical operational map of $F_{\mathrm{L}} / F_{\mathrm{D}}$ scaling factor $\sim$ particle blockage ratio for particle differential focusing patterns. The map is derived from the linear extrapolation of three existing particle blockage ratios.

Furthermore, we systematically tested inertial focusing of a wide range of particle sizes $(a=5-20 \mu \mathrm{m})$ in symmetric sinusoidal channels with different channel sizes $\left(D_{\mathrm{h}}=80-161 \mu \mathrm{m}\right.$, $\left.D_{\mathrm{h}} / 2 R=0.11-0.28\right)$ and aspect ratios $(H / W=0.167-0.55)$. We subsequently derived a universal experimental operational map of inertial differential focusing patterns based on the $F_{\mathrm{L}} / F_{\mathrm{D}}$ scaling factor $\delta=\left(a / D_{\mathrm{h}}\right) /\left[\mathrm{De}^{1 / 2}\left(D_{\mathrm{h}} / 2 R\right)^{3 / 4}\right]$, Figure 6 . Instead of directly using the particle blockage ratio $\left(a / D_{\mathrm{h}}\right)$ as the $x$-axis of the map, we modified the particle blockage ratio with the channel aspect ratio, $\left.\left(a / D_{\mathrm{h}}\right)\right) \times 2 /(1+H / W)$, which is equivalent of $a / H$. Two reasons justify this modification. First, the particle blockage ratio should not exceed 1 . Therefore, the ratio of particle diameter to channel height $a / H$ is more suitable to scale the relevant size of particle to channel cross-section in low-aspect-ratio channels $(H<W)$. Second, we found that the channel 
aspect ratio $H / W$ affects particle inertial focusing, and it should reflect in the operational map. After the modification using the aspect ratio, we found the best linear relationship and a clear threshold of $\delta$ for particle differential focusing patterns using the modified particle blockage ratio $\left(a / D_{\mathrm{h}}\right) \times 2 /(1+H / W)$ or $(a / H)$.

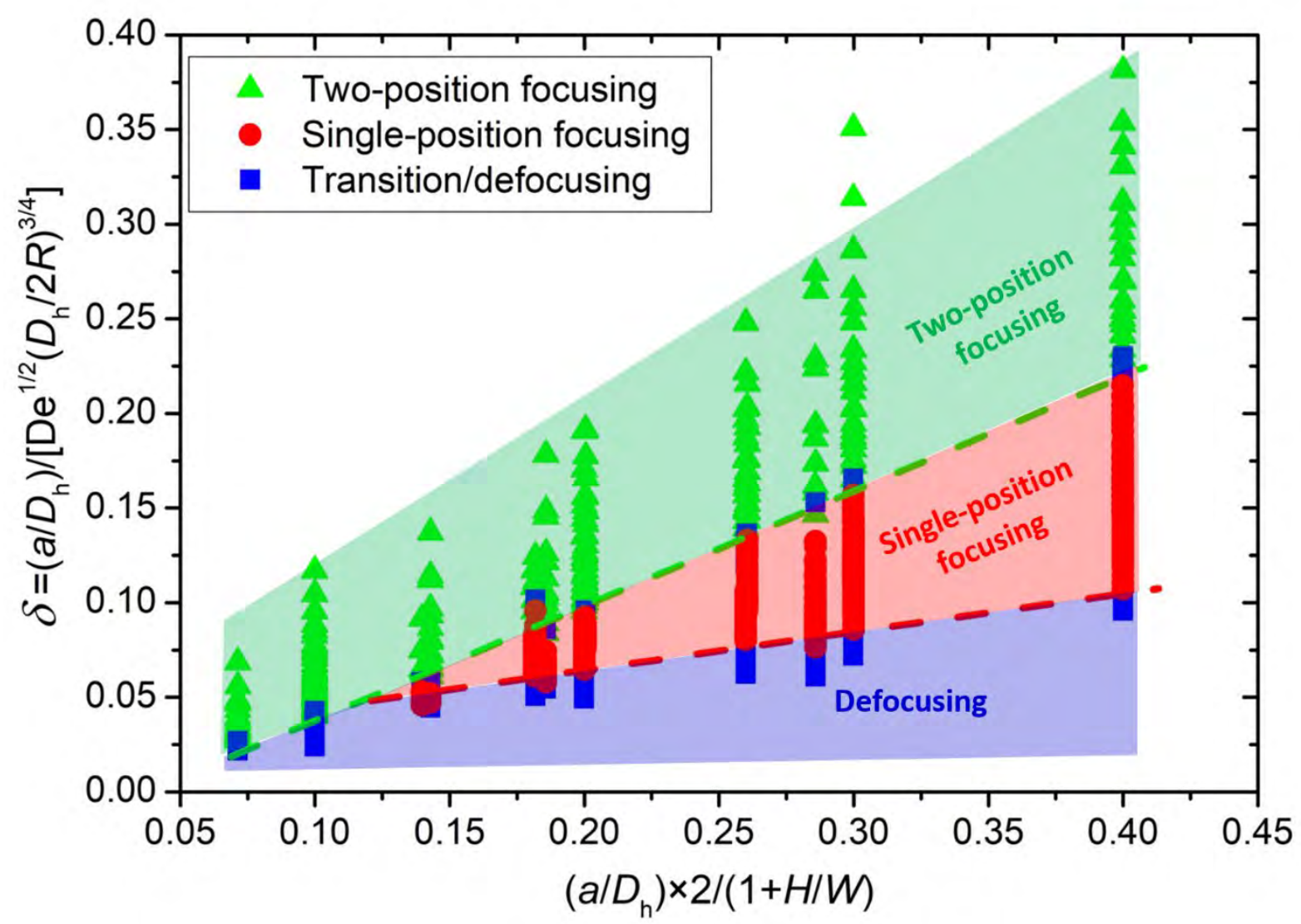

Figure 6 The experimental operational map of $F_{\mathrm{L}} / F_{\mathrm{D}}$ scaling factor $\delta=\left(a / D_{\mathrm{h}}\right) /\left[\mathrm{De}^{1 / 2}\left(D_{\mathrm{h}} / 2 R\right)^{3 / 4}\right]$ $\sim$ the modified particle blockage ratio $\left(a / D_{\mathrm{h}}\right) \times 2 /(1+H / W)$ or $(a / H)$ for particle differential focusing patterns.

Meanwhile, a rational design guide of sinusoidal microchannels for the separation of a specific binary particle mixture is proposed based on this operational map. Figure 7(a) depicts the flowchart for the design guidelines for symmetric sinusoidal channels. Particles should focus differentially along lateral directions in the microchannel, to achieve high-efficiency separation of binary particle mixture. Larger particles $a_{1}$ are assigned with a single-position focusing pattern at the channel centreline since they have a wider range of $\delta$ corresponding to single-position focusing pattern (Region II), while smaller particles $a_{2}$ should focus in two positions along the sidewalls (Region I), Figure 7(b). In this way, complete separation based 
on differential focusing patterns of binary particles can be obtained. Based on this design scheme, we designed and fabricated a sinusoidal microchannel for separation of a specific binary particle mixture, Figure S3. Moreover, when sorting a mixture of particles with multiple sizes ( $n \geq 3)$, various sinusoidal microchannels can be connected in a serial manner, and each sinusoidal microchannel sorts and purifies a specific range of particles.

(a)

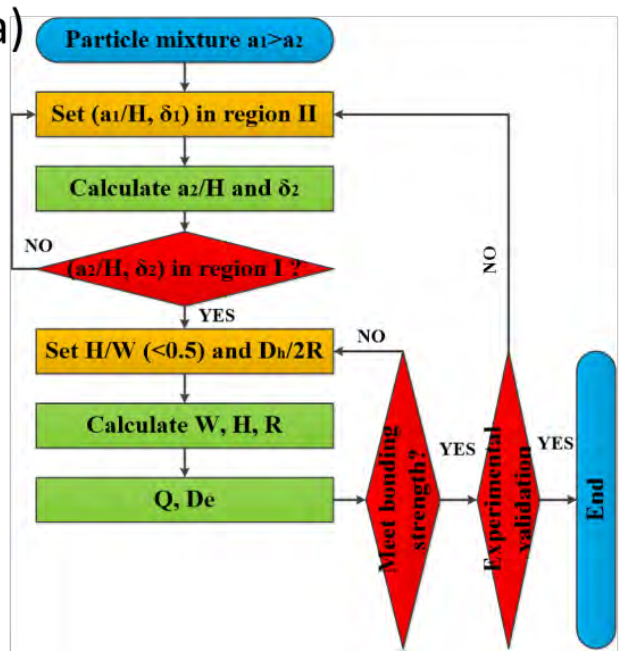

(b)

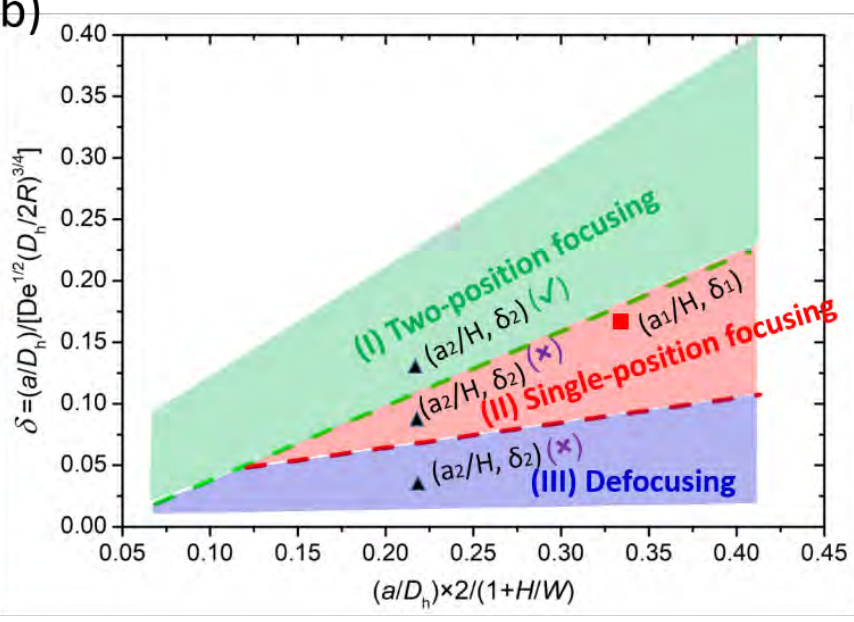

Figure 7 (a) The flowchart for designing symmetric sinusoidal microchannels for separation of a given binary particle mixture. (b) In order to achieve successful particle separation, two particles should focus as different patterns in the operational map. For larger particles $a_{1}$, they are preferably focused at the channel centreline as a single streak (Region II), while smaller particles $a_{2}$, they should focus along the two sidewalls (Region I). In this way, complete separation based on differential focusing patterns of binary particles mixture can be obtained.

\section{Conclusion}

The present paper systematically investigated particle inertial focusing phenomenon in lowaspect-ratio $(<1 / 2)$ symmetric sinusoidal microchannels. We comprehensively studied the effects of medium viscosity, particle size, flow condition and geometric dimension of microchannels on the particle differential focusing patterns. We observed that particle inertial focusing is independent of fluid viscosity, but highly dependent on particle size, flow condition and channel dimension as well as channel aspect ratio. We derived a dimensionless number $\delta=\left(a / D_{\mathrm{h}}\right) /\left[\mathrm{De}^{1 / 2}\left(D_{\mathrm{h}} / 2 R\right)^{3 / 4}\right]$ based on the ratio of inertial lift force $F_{\mathrm{L}}$ and Dean drag force $F_{\mathrm{D}}$. The value of $\delta$ is indicative for the particle differential focusing patterns, and it shows a high dependence on the relative dimension of particle to channel and channel aspect 
ratio $\left(a / D_{\mathrm{h}}\right) \times 2 /(1+H / W)$. A parametric map of particle differential focusing patterns was summarised based on the large collection of experimental data and theoretical analysis. The operational map of $\delta v s\left(a / D_{\mathrm{h}}\right) \times 2 /(1+H / W)$ intuitively takes in account the relationships of channel curvature ratio $\left(D_{\mathrm{h}} / 2 R\right)$, channel aspect ratio $(H / W)$, particle blockage ratio $\left(a / D_{\mathrm{h}}\right)$ and Dean number (De) for focusing patterns in symmetric sinusoidal microchannels. This operational map can help to predict the focusing pattern of specific microparticles as well as instruct design of sinusoidal microchannels for separation of a specific particle mixture.

\section{Conflicts of interest}

There are no conflicts of interest to declare.

\section{Supporting information}

Materials and methods, effects of medium kinetic viscosity, and continuous separation of microparticles based on differential focusing positions are in the supporting information.

Figure S1. A colour-coded map illustrates the quantitative distribution of particles along channel lateral direction at different flow conditions. The value of the colours in the map represents the normalized frequency of particles at the specific lateral position.

Figure S2 Inertial focusing of particles within glycerol aqueous solution with glycerol volume ratios ranging from $0 \%$ to $40 \%$.

Figure S3 Continuous separation of $10-\mu \mathrm{m}$ and $15-\mu \mathrm{m}$ particles based on differential focusing positions at the sinusoidal channel.

\section{Acknowledgements}

J. Zhang greatly acknowledges the support from the National Natural Science Foundation of China (Grant No.51705257) and the Natural Science Foundation of Jiangsu Province (Grant No. BK20170839). N.T. Nguyen and W.H. Li acknowledge the support from the Australian Research Council (ARC) Discovery Project (Grant No. DP180100055). S. Yan is a recipient of International Postdoctoral Fellowship of Japan Society for the Promotion of Science.

\section{References}


(1) Çetin, B.; Li, D. Dielectrophoresis in microfluidics technology. Electrophoresis. 2011, 32, 2410-2427.

(2) Wang, Z.; Zhe, J. Recent advances in particle and droplet manipulation for lab-on-a-chip devices based on surface acoustic waves. Lab Chip. 2011, 11, 1280-1285.

(3) Grier, D. G. A revolution in optical manipulation. Nature. 2003, 424, 810-816.

(4) Yamada, M.; Nakashima, M.; Seki, M. Pinched flow fractionation: continuous size separation of particles utilizing a laminar flow profile in a pinched microchannel. Anal. Chem. 2004, 76, 5465-5471.

(5) Huang, L. R.; Cox, E. C.; Austin, R. H.; Sturm, J. C. Continuous particle separation through deterministic lateral displacement. Science. 2004, 304, 987-990.

(6) Choi, S.; Song, S.; Choi, C.; Park, J. K. Sheathless focusing of microbeads and blood cells based on hydrophoresis. Small. 2008, 4, 634-641.

(7) Nguyen, N.-T.; Beyzavi, A.; Ng, K. M.; Huang, X. Kinematics and deformation of ferrofluid droplets under magnetic actuation. Microfluid. Nanofluid. 2007, 3, 571-579.

(8) Di Carlo, D. Inertial microfluidics. Lab Chip. 2009, 9, 3038-3046.

(9) Zhang, J.; Yan, S.; Yuan, D.; Alici, G.; Nguyen, N.-T.; Ebrahimi Warkiani, M.; Li, W. Fundamentals and applications of inertial microfluidics: a review. Lab Chip. 2016, 16, 10-34.

(10) Martel, J. M.; Toner, M. Inertial Focusing in Microfluidics. Annu. Rev. Biomed. Eng. 2014, 16, 371-396.

(11) Cristofanilli, M.; Budd, G. T.; Ellis, M. J.; Stopeck, A.; Matera, J.; Miller, M. C.; Reuben, J. M.; Doyle, G. V.; Allard, W. J.; Terstappen, L. W. M. M. Circulating tumor cells, disease progression, and survival in metastatic breast cancer. N. Engl. J. Med. 2004, 351, 781-791.

(12) Lee, M. G.; Choi, S.; Kim, H. J.; Lim, H. K.; Kim, J. H.; Huh, N.; Park, J. K. Inertial blood plasma separation in a contraction-expansion array microchannel. Appl. Phys. Lett. 2011, 98, 253702.

(13) Kuntaegowdanahalli, S. S.; Bhagat, A. A. S.; Kumar, G.; Papautsky, I. Inertial microfluidics for continuous particle separation in spiral microchannels. Lab Chip. 2009, 9, 2973-2980.

(14) Wu, L.; Guan, G.; Hou, H. W.; Bhagat, A. A. S.; Han, J. Separation of Leukocytes from Blood Using Spiral Channel with Trapezoid Cross-Section. Anal. Chem. 2012, 84, 9324-9331. (15) Sollier, E.; Amini, H.; Go, D.; Sandoz, P.; Owsley, K.; Di Carlo, D. Inertial microfluidic programming of microparticle-laden flows for solution transfer around cells and particles. Microfluid. Nanofluid. 2015, 19, 53-65.

(16) Gossett, D. R.; Tse, H. T. K.; Dudani, J. S.; Goda, K.; Woods, T. A.; Graves, S. W.; Di Carlo, D. Inertial manipulation and transfer of microparticles across laminar fluid streams. Small. 2012, 8, 2757-2764.

(17) Martel, J. M.; Smith, K. C.; Dlamini, M.; Pletcher, K.; Yang, J.; Karabacak, M.; Haber, D. A.; Kapur, R.; Toner, M. Continuous Flow Microfluidic Bioparticle Concentrator. Sci. Rep. 2015, 5, 11300.

(18) Hou, H. W.; Warkiani, M. E.; Khoo, B. L.; Li, Z. R.; Soo, R. A.; Tan, D. S.-W.; Lim, W.-T.; Han, J.; Bhagat, A. A. S.; Lim, C. T. Isolation and retrieval of circulating tumor cells using centrifugal forces. Sci. Rep. 2013, 3, Art No. 1259.

(19) Warkiani, M. E.; Guan, G.; Luan, K. B.; Lee, W. C.; Bhagat, A. A. S.; Chaudhuri, P. K.; Tan, D. S.-W.; Lim, W. T.; Lee, S. C.; Chen, P. C. Slanted spiral microfluidics for the ultrafast, label-free isolation of circulating tumor cells. Lab Chip. 2014, 14, 128-137.

(20) Ozkumur, E.; Shah, A. M.; Ciciliano, J. C.; Emmink, B. L.; Miyamoto, D. T.; Brachtel, E.; Yu, M.; Chen, P.-i.; Morgan, B.; Trautwein, J. Inertial focusing for tumor antigendependent and-independent sorting of rare circulating tumor cells. Sci. Transl. Med. 2013, 5, 179 ra147. 
(21) Warkiani, M. E.; Tay, A. K. P.; Khoo, B. L.; Xiaofeng, X.; Han, J.; Lim, C. T. Malaria detection using inertial microfluidics. Lab Chip. 2015, 15, 1101-1109.

(22) Nunes, J. K.; Wu, C. Y.; Amini, H.; Owsley, K.; Di Carlo, D.; Stone, H. A. Fabricating Shaped Microfibers with Inertial Microfluidics. Adv. Mater. 2014, 26, 3712-3717.

(23) Lee, W. C.; Bhagat, A. A. S.; Huang, S.; Van Vliet, K. J.; Han, J.; Lim, C. T. Highthroughput cell cycle synchronization using inertial forces in spiral microchannels. Lab Chip. 2011, 11, 1359-1367.

(24) Kemna, E. W.; Schoeman, R. M.; Wolbers, F.; Vermes, I.; Weitz, D. A.; van den Berg, A. High-yield cell ordering and deterministic cell-in-droplet encapsulation using Dean flow in a curved microchannel. Lab Chip. 2012, 12, 2881-2887.

(25) Gossett, D. R.; Henry, T.; Lee, S. A.; Ying, Y.; Lindgren, A. G.; Yang, O. O.; Rao, J.; Clark, A. T.; Di Carlo, D. Hydrodynamic stretching of single cells for large population mechanical phenotyping. Proc. Natl. Acad. Sci. U.S.A. 2012, 109, 7630-7635.

(26) Wang, L.; Dandy, D. S. High-Throughput Inertial Focusing of Micrometer-and Sub-Micrometer-Sized Particles Separation. Advanced Science. 2017, 4, 1700153.

(27) Zhou, Y.; Ma, Z.; Ai, Y. Sheathless inertial cell focusing and sorting with serial reverse wavy channel structures. Microsystems \& Nanoengineering. 2018, 4, 5.

(28) Di Carlo, D.; Irimia, D.; Tompkins, R. G.; Toner, M. Continuous inertial focusing, ordering, and separation of particles in microchannels. Proc. Natl. Acad. Sci. U.S.A. 2007, 104, 18892-18897.

(29) Gossett, D. R.; Carlo, D. D. Particle focusing mechanisms in curving confined flows. Anal. Chem. 2009, 81, 8459-8465.

(30) Di Carlo, D.; Jon, F.; Irimia, D.; Tompkins, R. G.; Toner, M. Equilibrium separation and filtration of particles using differential inertial focusing. Anal. Chem. 2008, 80, 2204-2211.

(31) Zhang, J.; Yan, S.; Li, W.; Alici, G.; Nguyen, N.-T. High throughput extraction of plasma using a secondary flow-aided inertial microfluidic device. RSC Advances. 2014, 4, 33149-33159.

(32) Zhang, J.; Li, W.; Li, M.; Alici, G.; Nguyen, N.-T. Particle inertial focusing and its mechanism in a serpentine microchannel. Microfluid. Nanofluid. 2014, 17, 305-316.

(33) Zhang, J.; Yan, S.; Sluyter, R.; Li, W.; Alici, G.; Nguyen, N.-T. Inertial particle separation by differential equilibrium positions in a symmetrical serpentine micro-channel. Sci. Rep. 2014, 4, Art No. 4527.

(34) Zhang, J.; Yuan, D.; Sluyter, R.; Yan, S.; Zhao, Q.; Xia, H.; Tan, S. H.; Nguyen, N.-T.; Li, W. High-Throughput Separation of White Blood Cells From Whole Blood Using Inertial Microfluidics. IEEE Transactions on Biomedical Circuits and Systems. 2017, 11, 1422-1430.

(35) Segre, G. Radial particle displacements in Poiseuille flow of suspensions. Nature. 1961, 189, 209-210.

(36) Segre, G.; Silberberg, A. Behaviour of macroscopic rigid spheres in Poiseuille flow Part 2. Experimental results and interpretation. J. Fluid Mech. 1962, 14, 136-157.

(37) ASMOLOV, E. S. The inertial lift on a spherical particle in a plane Poiseuille flow at large channel Reynolds number. J. Fluid Mech. 1999, 381, 63-87.

(38) Berger, S.; Talbot, L.; Yao, L. Flow in curved pipes. Annual review of fluid mechanics. 1983, 15, 461-512.

(39) Zhou, J.; Papautsky, I. Fundamentals of inertial focusing in microchannels. Lab Chip. 2013, 13, 1121-1132. 


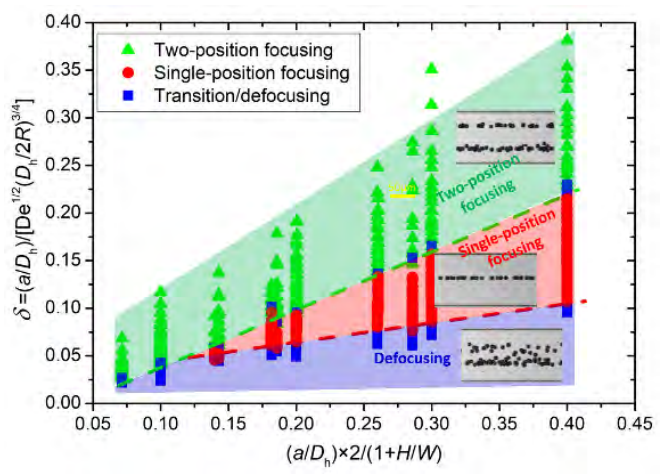

For TOC only 